\section{New resolve in inflammation}

\section{By Michael J. Haas, Senior Writer}

A report in Nature Immunology describes how the endogenous molecule resolvin E1 promotes resolution of airway inflammation in mice and provides a rationale for using this molecule as a therapeutic agent to treat asthma. Company and institution representatives interviewed for this story agreed that the findings could have multiple applications in the inflammation space. Opinions varied, however, on whether resolvin E1 was a potential therapeutic agent to treat asthma in humans.

The research team on the Nature Immunology paper was led by Bruce Levy, associate professor of medicine at Harvard Medical School and Brigham and Women's Hospital. ${ }^{1}$

Resolvins, a family of lipids produced in animal tissue from omega- 3 fatty acids, were first identified as key players in the resolution of inflammation in studies of murine abscesses published in 2000 and 2002 by teams at Harvard Medical School and Brigham and Women's Hospital., ${ }^{2,3}$ But until now their mechanism of action has remained unknown.

Levy said the Nature Immunology study built on previous work that found resolvin E1 (RvE1) plays a role in the resolution of acute inflammation. "The current work defines that mechanism," he said.

Indeed, Levy's group showed that RvE1 suppresses the proinflammatory IL-23 and IL-17 pathway and induces the anti-inflammatory molecules interferon- $\gamma\left(\right.$ IFN- $\gamma$ ) and lipoxin $\mathrm{A}_{4}\left(\mathrm{LXA}_{4}\right)$.

"In these experiments, Bruce, who is a pulmonologist, took the earlier, general findings about resolvins into the lungs and asthma, then tied them to the work we did on lipoxin $\mathrm{A}_{4}$, said Charles Serhan, coauthor on the paper.

Serhan, professor of anesthesia at Harvard Medical School and director of the Center for Experimental Therapeutics and Reperfusion Injury at Brigham and Women's Hospital, has helped discover both resolvins and lipoxins. .,3,, $6^{-6}$

\section{Allergic mice}

Levy's team used a mouse model they had developed for an earlier study of airway inflammation in asthma. ${ }^{7} \mathrm{He}$ said there are no animal models of the disease per se because asthma is strictly a human condition. Instead, models typically subject the animals' airways to allergens-a process known as airway challenge-to induce airway inflammation and/or airway hyper-responsiveness, which are two hallmarks of asthma in humans.

Airway inflammation involves constriction of the airways thought to be initiated by a $\mathrm{T}$ helper type 2 (Th2) immune response to airway challenge. Airway hyper-responsiveness is a twitching or spasmodic airway that can result in coughing, wheezing or shortness of breath.

Using the team's model, Levy said, "we stopped the challenge, allowed it to resolve spontaneously and tracked the mediators and counter-regulators of inflammation that are involved-IL-23, IL17, resolvin E1. This models the asthma response and resolution of inflammation that occurs in humans."

The team first administered intravenous RvE1 to the mice and then challenged their airways with an aerosol of ovalbumin, an egg protein commonly used to induce allergic responses. The RvE1-treated mice had significantly lower levels of eosinophils, leukocytes and macrophages in their lungs than did untreated mice, indicating that RvE1 had blocked the inflammatory response.

The team also showed that mice treated with intravenous RvE1 after airway challenge experienced faster resolution of inflammation than untreated mice.

"For this work we did track the time course to resolution of airway inflammation but not the resolution of airway hyper-responsiveness," Levy noted. "That will be reported in a forthcoming publication."

Next, the team examined serum, lung cells and bone marrow cells from the RvE1-treated mice. They found that RvE1 lowered the Th17 immune response and IL-17 levels by suppressing the production of IL-6 and IL-23. IL-17-producing T cells depend on those two cytokines for differentiation and survival.

Additionally, RvE1 increased production of the anti-inflammatory molecules IFN- $\gamma$ and $\mathrm{LXA}_{4}$ to accelerate resolution of the inflammation.

The team noted that RvE1 had no significant effect on either the Th2 response or the levels of proinflammatory IL-10, both of which are thought to be involved in the initiation of allergic inflammation. This result indicated that RvE1 acted selectively on the Th17 response, which maintains the inflammatory response even after exposure to allergens has ended.

Lastly, the airway-challenged mice received 15-epi-16-para-fluorophenoxy-LXA 4 methyl ester, an aspirin-triggered $\mathrm{LXA}_{4}$-stable analog (ATLa), alone and in combination with RvE1, and the team found that the two molecules contributed to resolution of airway inflammation in distinct ways. Both lowered levels of IL-17, but only RvE1 reduced levels of IL-23, indicating that RvE1 regulated IL-23 in a manner independent of $\mathrm{LXA}_{4}$.

Collectively, the findings suggest that RvE1 or its analogs could be new therapeutic agents to treat asthma. 
Levy noted that other work indicates RvE1 plays a role in the regulation of gastrointestinal mucosae and inflammatory bowel disease (IBD). ${ }^{8,9}$ "So RvE1 may regulate mucosal inflammation generally," he said.

\section{RvE1 reviews}

"The findings link RvE1 to the IL-23 and IL-17 pathway, which is involved in other inflammatory indications such as IBD, rheumatoid arthritis, corneal disease and plaque instability in atherosclerosis," said Per Gjorstrup, CMO and cofounder of Resolvyx Pharmaceuticals Inc., which has in-licensed the patent portfolio of resolvins and their mimics from Brigham and Women's Hospital. “Thus, the findings confirm the huge potential for resolvins for asthma and more generally in other inflammatory conditions."

Resolvyx has a synthetic form of RvE1, RX-10001, in preclinical development to treat asthma, colitis, rheumatoid arthritis (RA), atherosclerosis, dry eye and retinopathy. The compound has been formulated for i.v., subcutaneous and oral administration.

Gjorstrup said clinical trials of RX-10001 are expected to start in 2009, but the company has not yet decided which indication to pursue first. Meanwhile, Resolvyx plans to begin a Phase I trial of RX-10045, a synthetic resolvin analog, to treat dry eye in 3Q08.

"This is a very interesting paper that includes several novel aspects of allergic pulmonary inflammation," said Charlotte Edenius, CSO and VP of preclinical development at Orexo AB. "The effects of RvE1 demonstrated in this paper definitely suggest a potential for new treatments for asthma and possibly other respiratory inflammatory disorders," she said.

\section{"There is very limited, if any, knowledge on the resolution of inflammation in allergic respiratory disease, and the role of RvE1 is intriguing." -Charlotte Edenius, Orexo AB}

suggested-but did not prove-that RvE1 similarly suppressed IL-23 and IL-6 directly in the mouse lung. "It is questionable whether the concentration of resolvin E1 in the lung reaches the concentration required to show inhibition of the cytokines in vitro," she said.

Levy told SciBX that the team observed concentration-dependent effects of RvE1 in vitro up to about $3 \mathrm{nM}$. Although the researchers did not specifically measure the concentrations of RvE1 in the mouse lung, he said that RvE1 appeared to be more potent in vivo than in vitro, suggesting that it acted at multiple sites or on multiple cellular targets.

Like Orexo's Edenius, Theodore Reiss, VP of clinical research in the Department of Respiratory, Allergy, Gastrointestinal and Urologic Diseases at Merck \& Co. Inc.'s Merck Research Laboratories, said the findings were an important advance in understanding asthma. But like Wada, he expressed caution about whether RvE1 could treat the disease.

"Whether airway inflammation in rodents reflects asthma in humans is controversial," Reiss told SciBX. "Nevertheless, it has recently been suggested that the Th17 pathway may play a role in asthma, and this study provides further evidence for this hypothesis by demonstrating that modulation of this system has an effect on allergic inflammation in rodents."

Levy acknowledged that some researchers think larger animals-sheep, in particularmight make better models of asthma, but he had no direct experience using them.

"There are some similarities between human and sheep in terms of lung anatomy, development and electrophysiology," he said.

Levy also enumerated several other advantages: sheep are similar in body weight to humans; their lung functions can be measured while they are conscious, as is done in human

Edenius agreed with Gjorstrup and Levy that the findings have implications beyond respiratory disease, given the broad proinflammatory effects of IL-23 and IL-17.

Orexo is developing two classes of anti-inflammatory compounds15-lipoxygenase inhibitors and eoxin inhibitors-to treat respiratory inflammatory indications such as asthma, chronic obstructive pulmonary disease (COPD) and rhinitis. The enzyme 15-lipoxygenase catalyzes the conversion of arachidonic acid, an omega- 6 fatty acid, into proinflammatory eoxins.

Yumiko Wada, senior director of biology at Synta Pharmaceuticals Inc., also said the Nature Immunology findings could apply to other diseases in which the IL-23 and IL-17 pathway has been implicated, such as RA, IBD, psoriasis and multiple sclerosis (MS). "The blockade of IL-23, a critical upstream trigger, could be an effective therapeutic strategy in any of these diseases," she said.

Wada added that RvE1 has been shown to promote the resolution of dermal inflammation, peritonitis and colitis in murine models and said the fact that RvE1 blocks the Th17 inflammatory response but not the Th2 response could be a therapeutic advantage. "The selective inhibitory activity of resolvin E1 on the IL-23 and Th17 pathway that drives many inflammatory diseases is ideal for the treatment of those diseases," she said.

But Wada was cautious about RvE1's potential to treat asthma, noting that the suppression of IL- 23 and IL- 6 by RvE1 in vitro only patients; and multiple tissue samples can be taken from the same animal.

But he added that sheep models are expensive and time consuming, and the limited availability of immunological and molecular tools to study them are disadvantages to their use.

\section{Breathing strategies}

In addition to questions about whether RvE1 acts in humans as it does in mice, there was no consensus on whether inhibiting inflammation or promoting the resolution of inflammation is the better approach in asthma.

Edenius noted that promoting resolution of inflammation with an agonist like RvE1 - rather than inhibiting it with an anti-inflammatory drug - is a new approach to treating asthma that requires further proof of concept.

"There is very limited, if any, knowledge on the resolution of inflammation in allergic respiratory disease, and the role of RvE1 is intriguing," she said. "I believe we just have to wait for more supportive data before knowing whether this will be another tool for treatment of a large disease with great medical need."

Merck's Reiss agreed that the idea of treating asthma by promoting resolution of inflammation needed more validation. "This is a very early idea," he said. "The effects in other model systems would need to be assessed, and its in vivo biologic relevance in humans would need 


\section{TARGETS \& MECHANISMS}

to be assessed, especially in populations where it might be biologically important."

For instance, Reiss said, RvE1's mechanism might be relevant in patients with severe asthma, where leukocytes like neutrophils and eosinophils might have a greater pathological impact.

Although further studies are needed to flesh out the viability of an inflammation resolution strategy, the findings on RvE1 do raise important questions for other approaches to treating asthma and other inflammatory indications. A case in point, said Edenius, are inhibitors of 5-lipoxygenase or 5-lipoxygenase activating protein (FLAP).

FLAP activates 5-lipoxygenase, which converts arachidonic acid into leukotrienes, a family of proinflammatory molecules. But Edenius noted that these inhibitors also block the formation of RvE1 and lipoxins. Thus, she suggested, the Nature Immunology paper "indicates that it might be better to block leukotriene synthesis downstream of the 5-lipoxygenase and FLAP step, and thereby preserve RvE1 and lipoxin formation."

\section{Resolving specifics}

Going forward, Levy said the team plans to investigate specific aspects of RvE1's role in resolving inflammation.

One is the mechanism by which downregulation of the Th17 response facilitates that resolution. Another is the specific relationships between RvE1, IFN- $\gamma$ and $\mathrm{LXA}_{4}$ in resolving inflammation.

"LXA is also upregulated naturally during resolution, and RvE1 further increases it," Levy said. "This suggests that RvE1 is upstream of LXA $_{4}$. Yet there are some distinct mechanisms for each of these molecules in counter-regulating inflammation. We want to clarify this relationship."

Conversely, Levy noted that like cystic fibrosis patients, the severe asthma population is characterized by a decreased ability to produce $\mathrm{LXA}_{4}$.

"We don't know about RvE1 in that context," he said. "But it's certainly provocative to wonder whether the ability to produce RvE1 is also reduced in these patients."

\section{REFERENCES}

1. Haworth, O. et al. Nat. Immunol.; published online June 22, 2008; doi:10.1038/ni.1627

Contact: Bruce Levy, Brigham and Women's Hospital and Harvard Medical School, Boston, Mass. e-mail: blevy@partners.org

2. Serhan, C. et al. J. Exp. Med. 192, 1197-1204 (2000)

3. Serhan, C. et al. J. Exp. Med. 196, 1025-1037 (2002)

4. Schwab, J. et al. Nature 447, 869-874 (2007)

5. Serhan C. et al. Biochem. Biophys. Res. Commun. 118, 943-949 (1984)

6. Serhan, C. et al. Proc. Natl. Acad. Sci. USA 81, 5335-5339 (1984)

7. Levy, B. et al. J. Immunol. 178, 496-502 (2007)

8. Arita, M. et al. Proc. Natl. Acad. Sci. USA 102, 7671-7676 (2005)

9. Hudert, C. et al. Proc. Natl. Acad. Sci. USA 103, 11276-11281 (2006)

\section{COMPANIES AND INSTITUTIONS MENTIONED}

Brigham and Women's Hospital, Boston, Mass. Harvard Medical School, Boston, Mass.

Merck \& Co. Inc. (NYSE:MRK), Whitehouse Station, N.J.

Orexo AB (SSE:ORX), Uppsala, Sweden

Resolvyx Pharmaceuticals Inc., Bedford, Mass.

Synta Pharmaceuticals Inc. (NASDAQ:SNTA), Lexington, Mass. 